\title{
Childhood Gemistocytic Astrocytoma
}

National Cancer Institute

\section{Source}

National Cancer Institute. Childhood Gemistocytic Astrocytoma. NCI Thesaurus. Code C114964.

A gemistocytic astrocytoma that occurs during childhood. 